\title{
Diagnóstico tardío de síndrome de interrupción del tallo hipofisario
}

\author{
Vanessa Garzón Graciano ${ }^{1}$, Santiago González Narvaez ${ }^{1}$, Jhon Alexander Alzate Piedrahita², \\ Víctor Hugo Ruiz Granada ${ }^{3}$, Santiago Vallejo González $z^{4}$
}

\begin{abstract}
${ }^{1}$ Estudiantes de Medicina Universidad Tecnológica de Pereira. ${ }^{2}$ Médico internista Hospital Universitario San Jorge de Pereira. Docente Universidad Tecnológica de Pereira.

${ }^{3}$ Médico radiólogo Imágenes Diagnósticas Hospital Universitario San Jorge de Pereira.

${ }^{4}$ Médico internista endocrinólogo Hospital Universitario San Jorge de Pereira. Docente Universidad Tecnológica de Pereira.
\end{abstract}

Autor responsable de correspondencia: Santiago Vallejo González. Dirección: Carrera 4 \# 20-55. Pereira, Risaralda. Celular: 3104037662. Correo electrónico: santivallejog@hotmail.com.

Declaración de fuentes de financiación y posibles conflictos de interés: Este trabajo no requirió ninguna inversión por lo que no tuvo ninguna fuente de financiación.

Para este reporte, ninguno de los autores posee conflictos de interés.

El Dr. Santiago Vallejo González ha recibido honorarios por conferencias en el área de diabetes por parte de NovoNordisk y Astra Zeneca y ha recibido apoyo económico para la asistencia a cursos y congresos por parte NovoNordisk, Amgen, Abbott, Lilly, Sanofi, Merck y Medtronic.

Fecha de recepción: 18/06/2017

Fecha de aceptación: 2/08/2017

\section{Resumen}

El síndrome de interrupción del tallo hipofisario (PSIS, por sus siglas en inglés) es una anomalía congénita de probable origen genético causante de hipopituitarismo, que se caracteriza por hipoplasia de la adenohipófisis, neurohipófisis ectópica e interrupción del tallo hipofisario. Suele presentarse con baja estatura y deficiencia adenohipofisaria. El retraso en el diagnóstico se relaciona con alta morbimortalidad. Se reporta el caso de un hombre de 39 años con fractura de cadera por fragilidad y posterior compromiso neurológico secundario a hiponatremia severa, hipoglucemia e hipotensión arterial. Presentaba hábito eunucoide y ausencia de caracteres sexuales secundarios, con genitales infantiles. Los paraclínicos mostraron compromiso de todas las hormonas adenohipofisarias, sin afección neurohipofisaria. Una resonancia magnética nuclear mostró hallazgos consistentes con PSIS.
Palabras clave: Hipopituitarismo, síndrome de interrupción del tallo hipofisario, hipogonadismo, retardo en el crecimiento, hiponatremia.

\section{Abstract}

The pituitary stalk interruption syndrome (PSIS) is a congenital defect with a possible genetic origin which causes hypopituitarism. It consists of anterior pituitary hypoplasia, ectopic posterior pituitary and interruption of the pituitary stalk. Its clinical presentation consists mainly in short stature and anterior pituitary hormonal deficiencies. The delayed diagnosis may cause morbidity and mortality. We report the case of a 39 year old male who presented with a frailty leg fracture and neurologic involvement due to severe hyponatremia, hypoglycemia and hypotension. He lacked secondary sexual development. Laboratory examinations showed an inadequate secretion of all anterior pituitary hormones without diabetes insipidus. A magnetic resonance image showed findings consistent with PSIS.

Keywords: Hypopituitarism, pituitary stalk interruption syndrome, hypogonadism, growth retardation, hiponatremia.

\section{Introducción}

El hipopituitarismo, definido como un déficit total o parcial de la función hipofisaria, es una enfermedad endocrina crónica que se ha asociado con aumento en la morbilidad y mortalidad, por lo que su diagnóstico temprano y el tratamiento oportuno son muy importantes. Las principales causas de hipopituitarismo incluyen tumores hipofisarios y secuelas de cirugía e irradiación a nivel pituitario. De forma menos frecuente, la etiología puede ser infecciosa, infiltrativa o autoinmune ${ }^{(1)}$.

El síndrome de interrupción del tallo hipofisario (PSIS) es un ejemplo de disrupción funcional de la unidad hipotálamohipófisis anterior, siendo una causa de deficiencia hormonal hipofisaria aislada o múltiple poco frecuente ${ }^{(2,3)}$.

Reportado por primera vez por Fujisawa et $\mathrm{al}^{(4)}$ se caracteriza por la triada clásica de tallo hipofisario interrumpido, hipófisis posterior ausente o ectópica e hipoplasia o aplasia de la hipófisis anterior. A continuación, presentamos el caso de 
un paciente con hipopituitarismo secundario a PSIS quien, de forma llamativa, tuvo un diagnóstico a una edad muy superior a la usualmente reportada en la literatura.

\section{Presentación del caso}

Paciente masculino de 39 años con antecedente de parto podálico, quien presentó fractura de cadera con trauma de bajo impacto y, adicionalmente, alteración del estado de conciencia. Ingresó al servicio de urgencias con una presión arterial de 100/60 mmHg, frecuencia cardíaca de 61 latidos por minuto y glucometría de $60 \mathrm{mg} / \mathrm{dl}$. Se notó estuporoso, con importante xerodermia y con hábito eunucoide. Pesó 48 kg y midió $160 \mathrm{~cm}$. Sus genitales eran infantiles, con presencia de micropene y testículos menores de $3 \mathrm{cc}$ de forma bilateral. Dentro de los estudios que se efectuaron al ingreso, se encontró un sodio sérico en $97 \mathrm{mEq} / \mathrm{L}$.

Dadas la fractura por fragilidad, la ausencia de desarrollo sexual, la hiponatremia, la hipoglucemia y la presión arterial limítrofe, se hicieron estudios hormonales (tabla 1).

Tabla 1. Paraclínicos hormonales

\begin{tabular}{l|c|c}
\multicolumn{1}{c|}{ Paraclínicos } & Valor & Referencia \\
\hline TSH & $1,5 \mathrm{mcUl} / \mathrm{mL}$ & $0,46-4,68 \mathrm{mcU} / \mathrm{mL}$ \\
\hline T4L & $0,41 \mathrm{ng} / \mathrm{dL}$ & $0,78-2,19 \mathrm{ng} / \mathrm{dL}$ \\
\hline Cortisol $8 \mathrm{am}^{*}$ & $6,2 \mathrm{mcg} / \mathrm{dL}$ & $>18 \mathrm{mcg} / \mathrm{dL}$ \\
\hline ACTH & $<1,6 \mathrm{pg} / \mathrm{ml}$ & $7-57 \mathrm{pg} / \mathrm{ml}$ \\
\hline Testosterona total & $0,01 \mathrm{ng} / \mathrm{ml}$ & $2,4-10,8 \mathrm{ng} / \mathrm{mL}$ \\
\hline FSH & $0,7 \mathrm{mUI} / \mathrm{mL}$ & $1,7-12 \mathrm{mUI} / \mathrm{mL}$ \\
\hline LH & $0,2 \mathrm{mUl} / \mathrm{mL}$ & $1,1-7,0 \mathrm{mUI} / \mathrm{mL}$ \\
\hline IGF-1 & $3 \mathrm{ng} / \mathrm{mL}$ & $98-396 \mathrm{ng} / \mathrm{mL}$ \\
\hline PRL & $8 \mathrm{ng} / \mathrm{mL}$ & $4,04-15,2 \mathrm{ng} / \mathrm{mL}$ \\
\hline Densidad Urinaria & $1.014 \mathrm{~g} / \mathrm{L}$ & $1.000-1.030 \mathrm{~g} / \mathrm{L}$ \\
\hline
\end{tabular}

*La muestra de cortisol fue tomada durante un episodio de hipoglucemia.

Ante el diagnóstico bioquímico de panhipopituitarismo, se obtuvo una resonancia magnética nuclear (RMN) de silla turca simple y con Gadolinio (figura 1), que mostró ectopia hipofisaria posterior con neurohipófisis ubicada hacia la base del tallo hipofisario, el cual lucía displásico y filiforme, asociado a adenohipófisis hipoplásica, configurando un síndrome de interrupción del tallo hipofisario. Adicionalmente, presentaba displasia de los cuerpos mamilares y del túber cinereum.

\section{Discusión}

El PSIS es una patología hipofisaria infrecuente (incidencia estimada de 0,5/1.000.000 de nacidos vivos) ${ }^{(5)}$ que se caracteriza por la alteración en los ejes adenohipofisarios y los hallaz- gos radiológicos característicos mencionados previamente. En Colombia existe solo un reporte de caso previo en un paciente de edad pediátrica ${ }^{(6)}$.

En neonatos, suele presentarse como hipoglucemia neonatal, ictericia fisiológica prolongada, criptorquidia y micropene; mientras que la baja estatura es la principal manifestación en infantes mayores y adultos. También se puede presentar como

Figura 1. Ectopia hipofisaria posterior con neurohipófisis ubicada a nivel del tallo hipofisario, el cual luce displásico y filiforme, asociado a adenohipófisis hipoplásica. Displasia de los cuerpos mamilares y del túber cinereum. RMN de silla turca simple y con gadolinio, corte sagital y coronal

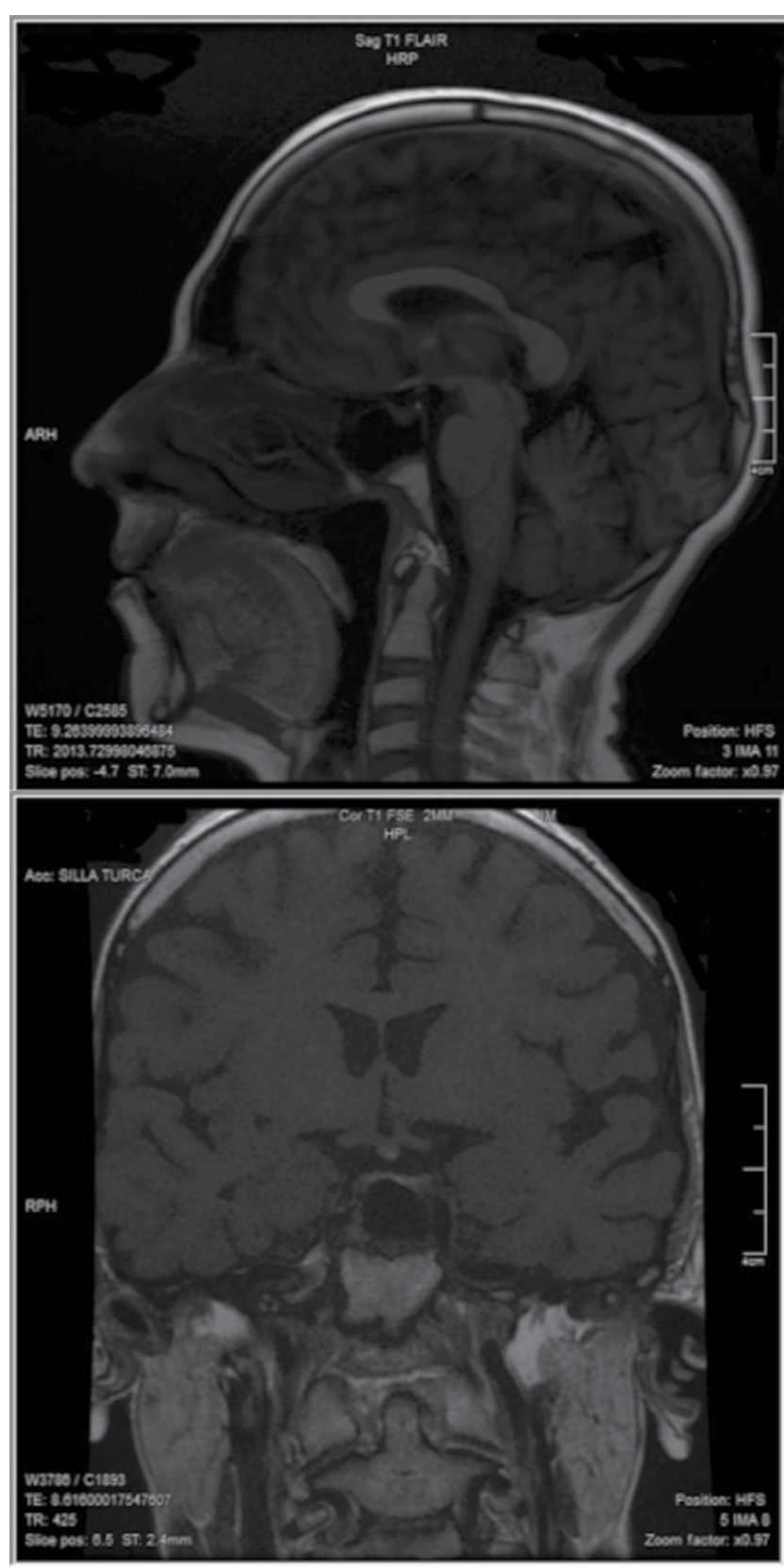


retraso en la pubertad o con alteraciones metabólicas como hipoglucemia e insuficiencia adrenal ${ }^{(2,5-7)}$.

En la literatura se reporta déficit de hormona de crecimiento en el $100 \%$ de los casos, de gonadotropinas en un $95,8 \%$, de corticotropina en un $81,1 \%$, y de tirotropina en un $76,3 \%$; adicionalmente, hiperprolactinemia en un $36,4 \%$ de los pacientes, aunque muchos pueden cursar con niveles bajos de prolactina ${ }^{(8)}$. En el 92,7\% de los casos se encontró déficit de al menos 2 hormonas hipofisarias ${ }^{(9)}$. Los hallazgos de la literatura se correlacionan con los del paciente, ya que éste presentó disfunción de todos los ejes adenohipofisarios, salvo la hiperprolactinemia que estuvo ausente.

La literatura indica que la etapa de la vida en la que se suele hacer el diagnóstico de PSIS es la infancia, siendo infrecuente a edades tardías, como ocurrió en el caso presentado.

El compromiso de la secreción hormonal en estos casos no siempre es simultáneo de todos los ejes hipofisarios. Se ha descrito que puede haber un compromiso evolutivo e inicialmente parcial que puede progresar en el tiempo. Lo anterior podría explicar una sintomatología larvada en ciertos pacientes y el retraso en el diagnóstico ${ }^{(10)}$.

Se ha considerado que la ausencia de tallo hipofisario se asocia con déficits múltiples y más severos, en comparación con los casos en los que se logra identificar un tallo, así éste sea anormal ${ }^{(3,8)}$.

La presentación clínica del síndrome depende de la edad en la que se haga el diagnóstico. En adultos, suele cursar con talla baja (en un $85 \%$ de los casos) ${ }^{(5)}$ y con ausencia de desarrollo de caracteres sexuales secundarios. El paciente presentaba hipogonadismo clínico, lo cual debió hacer sospechar de manera temprana alteraciones hormonales, con el consecuente estudio etiológico. Llamativamente, no tenía talla baja, lo que hace pensar en un compromiso tardío del eje somatotropo.
El diagnóstico se confirma mediante RMN. Los hallazgos radiológicos pueden incluir hipoplasia o aplasia de la adenohipófisis, ausencia de la hiperintensidad de la neurohipófisis a nivel de la silla turca y su presencia en la eminencia media en el sitio del tallo hipofisario y un infundíbulo delgado, ausente o interrumpido. La fisiopatología exacta de esta condición no está completamente aclarada, pero se ha encontrado una mayor frecuencia en pacientes con partos traumáticos, podálicos, con requerimiento de cesárea o ante hipoxia neonatal, aunque actualmente se considera a estas condiciones más como una consecuencia que como una causa de la afección ${ }^{(8)}$. La asociación del cuadro con micropene, criptorquidia y condiciones sindrómicas y familiares, ha hecho pensar que se debe a una condición antenatal. Se han reportado alteraciones en genes implicados en el desarrollo hipofisario, entre ellos HESX1, LHX4, SOX3 y PROKR2 ${ }^{(6,7)}$. Entre el 20 y el 50\% de los pacientes cursan con malformaciones congénitas asociadas y son particularmente frecuentes en personas con déficit aislado de hormona de crecimiento ${ }^{(7)}$.

\section{Conclusiones}

El PSIS es una condición asociada con disfunción hipofisaria que, aunque rara, debe ser considerada como diagnóstico diferencial ante una disfunción hipofisaria múltiple o aislada de hormona del crecimiento.

Hallazgos como déficit en el crecimiento y pubertad retardada deben orientar al médico a la realización oportuna de estudios de función hormonal y, de acuerdo con estos, valoración anatómica de la hipófisis con RNM en busca de la etiología.

El diagnóstico tardío y la falta de suplencia hormonal oportuna pueden relacionarse con complicaciones por el déficit hormonal crónico generando así una importante morbilidad e incluso condicionar mortalidad.

\section{Referencias}

1. Kim SY. Diagnosis and Treatment of Hypopituitarism. Endocrinol Metab (Seoul, Korea) [Internet]. 2015 Dec [cited 2017 Jun 14];30(4):443-55. Available from: http://www.ncbi.nlm.nih.gov/pubmed/26790380

2. Kulkarni C, Moorthy S, Pullara SK, Rajeshkannan R, Unnikrishnan AG. Pituitary stalk transection syndrome: Comparison of clinico-radiological features in adults and children with review of literature. Indian J Radiol Imaging [Internet]. 2012 Jul [cited 2017 Jun 14];22(3):182-5. Available from: http://www.ncbi.nlm.nih.gov/pubmed/23599565

3. Bordallo MAN, Tellerman LD, Bosignoli R, Oliveira FFRM, Gazolla FM, Madeira IR, et al. Neuroradiological investigation in patients with idiopathic growth hormone deficiency. J Pediatr (Rio J) [Internet]. 2004 May 15 [cited 2017 Jun 14];80(3):223-8. Available from: http://www.jped.com.br/Redirect.aspx?varArtigo $=1187$

4. Fujisawa I, Kikuchi K, Nishimura K, Togashi K, Itoh K, Noma S, et al. Transection of the pituitary stalk: development of an ectopic posterior lobe assessed with MR imaging. Radiology [Internet]. 1987 Nov [cited 2017 Jun 14];165(2):487-9. Available from: http://www.ncbi.nlm.nih.gov/ pubmed/3659371

5. Gutch M, Kumar S, Razi SM, Saran S, Gupta KK. Pituitary stalk interruption syndrome: Case report of three cases with review of literature. J Pediatr Neurosci [Internet]. 2014 May [cited 2017 Jun 14];9(2):188-91. Available from: http://www.ncbi.nlm.nih.gov/pubmed/25250085

6. Agudelo S, Maldonado MJ, Tovar X, Salinas CM. Panhipopituitarismo con- génito secundario a interrupción del tallo hipofisario: informe de caso. Rev Colomb Enfermería [Internet]. 2015 [cited 2017 Jun 14];10(10):90-4. Available from: http://m.uelbosque.edu.co/sites/default/files/publicaciones/revistas/revista_colombiana_enfermeria/volumen10/010_articulo8-Vol10 A10.pdf

7. Ram N, Ali SA, Hussain SZ. Pituitary stalk interruption syndrome presenting as short stature: a case report. J Med Case Rep [Internet]. 2014 Dec 19 [cited 2017 Jun 14];8(1):445. Available from: http://jmedicalcasereports. biomedcentral.com/articles/10.1186/1752-1947-8-445

8. Wang Q, Hu Y, Li G, Sun X. Pituitary stalk interruption syndrome in 59 children: the value of MRI in assessment of pituitary functions. Eur J Pediatr [Internet]. 2014 May 21 [cited 2017 Jun 14];173(5):589-95. Available from: http://www.ncbi.nlm.nih.gov/pubmed/24257915

9. Guo Q, Yang Y, Mu Y, Lu J, Pan C, Dou J, et al. Pituitary Stalk Interruption Syndrome in Chinese People: Clinical Characteristic Analysis of 55 Cases. Luque RM, editor. PLoS One [Internet]. 2013 Jan 14 [cited 2017 Jun 14];8(1):e53579. Available from: http://www.ncbi.nlm.nih.gov/ pubmed/23341953

10. Marmouch H, Graja S, Arfa S, Boubaker F, Khochtali I. [Late-onset pituitary stalk interruption syndrome (PSIS)]. Pan Afr Med J [Internet]. 2016 [cited 2017 Jun 14];23:108. Available from: http://www.ncbi.nlm.nih.gov/ pubmed/27231511 